\title{
Analysis of Histologic Parameters Associated with Nuclear Size in Diffuse Malignant Peritoneal Mesothelioma Uniformly Treated by Cytoreductive Surgery and Perioperative Intraperitoneal Chemotherapy
}

\section{Tristan D. Yan ${ }^{1}$, Erwin A. Brun², Carlos A. Cerruto ${ }^{3}$, David Chang ${ }^{4}$ and Paul H. Sugarbaker ${ }^{1 *}$}

${ }^{1}$ Washington Cancer Institute, Washington DC, USA

${ }^{2}$ Capital Digestive Care, Pathology Division, Bowie, MD, USA

${ }^{3}$ Dermpath Diagnostics, Altamonte Springs, FL, USA

${ }^{4}$ Westat Rockville, MD, USA

\begin{abstract}
Aims: Nuclear size has been identified as a significant prognostic indicator for survival after cytoreductive surgery and perioperative intraperitoneal chemotherapy for diffuse malignant peritoneal mesothelioma (DMPM). The additional histopathologic features associated within the clinical material have not been determined. This current study assessed the correlations between nuclear size and 12 other histopathologic parameters.

Methods: A review of the histopathological features of DMPM in 62 patients who underwent uniform management of cytoreductive surgery and perioperative intraperitoneal chemotherapy was performed. Nuclear size was categorized into two groups: $\leq 30 \mu . m(n=35)$ versus $>30 \mu . m(n=27)$. The correlations between nuclear size and 12 histopathologic parameters of DMPM were determined by univariate analysis.

Results: Patients with nuclear size $>30 \mu . \mathrm{m}$ had a less favorable prognosis, as compared to patients with nuclear size $\leq 30 \mu . \mathrm{m}(p<.001)$. Nuclear size was statistically correlated with histologic type $(p=.012)$, nuclear/cytoplasmic ratio $(p<.001)$, mitotic count $(p=.001)$, atypical mitosis $(p<.001)$, tumor necrosis $(p<.001)$, chromatin pattern $(p<$ $.001)$ and nucleolar size $(p<.001)$.

Conclusions: Histopathologic features present along with nuclear size are histologic type, nuclear/cytoplasmic ratio, mitotic count, atypical mitosis, tumor necrosis, chromatin pattern and nucleolar size in patients undergoing cytoreductive surgery and perioperative intraperitoneal chemotherapy. The identification of these histopathologic features may function as an adjunct to nuclear size in the estimation of prognosis.
\end{abstract}

Keywords: Peritoneal mesothelioma; Cytoreductive surgery; Intraperitoneal chemotherapy; Nuclear grading

\section{Introduction}

Several studies have documented that DMPM consists of heterogeneous histopathologic features [1-3]. In 1995 Goldblum and Hart first proposed a mesothelioma nuclear grading system which categorized the histomorphology of DMPM into four grades: Grade 0 represented small nuclei, uniform chromatin pattern and inconspicuous or absent nucleoli; Grade 1 represented small nuclei, uniform chromatin pattern and small pinpoint-sized nucleoli; Grade 2 represented large nuclei, some chromatin irregularity and more prominent nucleoli; and Grade 3 represented large nuclei, irregular chromatin pattern with clearing and prominent macronucleoli [3]. However, the number of patients studied did not allow a statistical assessment of the grading system to test its clinical value. Recently, Nonaka and co-workers demonstrated an improved survival in patients with low-grade nuclei after cytoreductive surgery and perioperative intraperitoneal chemotherapy [4]. Cerruto and colleagues used a group of 62 DMPM patients treated by cytoreductive surgery and perioperative intraperitoneal chemotherapy to search for histologic parameters that correlated with prognosis [5]. In this study they identified nuclear/nucleolar size as the most reliable single parameter by which to predict outcome. The current study attempts to identify other histologic features that can act as an adjunct to nuclear size in the histopathologic estimation of prognosis in DMPM patients to be treated by cytoreductive surgery and perioperative intraperitoneal chemotherapy.

\section{Patients and Methods}

\section{Patient Selection}

Sixty-two consecutive patients with a diagnosis of DMPM were treated in a uniform fashion utilizing cytoreductive surgery combined with perioperative intraperitoneal chemotherapy by the same surgical team at the Washington Cancer Institute, Washington, DC. These 62 patients formed the basis of this report. The clinical data were obtained prospectively and entered regularly into an electronic database by a research assistant. All patients were followed prospectively until the last time of contact or death.

\section{Preoperative Management}

All patients underwent extensive preoperative evaluations, including physical examination, abdominal, pelvic and chest CT to assess the extent of the disease. Measurements of relevant tumor markers (e.g., CA 15-3, CA 19-9, CA 72-4, CA 125 and Carcinoembryonic antigen) were also obtained.

\section{Cytoreductive Surgery}

The details of the surgical techniques have been described

*Corresponding author: Paul H. Sugarbaker, Washington Cancer Institute, 106 Irving Street, NW, Suite 3900, Washington DC, USA, Tel: 202-877--3908; Fax 202-877-8602; E-mail: Paul.Sugarbaker@medstar.net

Received December 07, 2011; Accepted February 02, 2012; Published February 10,2012

Citation: Yan TD, Brun EA, Cerruto CA, Chang D, Sugarbaker PH (2012) Analysis of Histologic Parameters Associated with Nuclear Size in Diffuse Malignan Peritoneal Mesothelioma Uniformly Treated by Cytoreductive Surgery and Perioperative Intraperitoneal Chemotherapy. Surgery S8:001. doi:10.4172/21611076.S8-001

Copyright: @ 2011 Yan TD, et al. This is an open-access article distributed under the terms of the Creative Commons Attribution License, which permits unrestricted use, distribution, and reproduction in any medium, provided the original author and source are credited. 

Malignant Peritoneal Mesothelioma Uniformly Treated by Cytoreductive Surgery and Perioperative Intraperitoneal Chemotherapy. Surgery S8:001. doi:10.4172/2161-1076.S8-001

Page 2 of 5

elsewhere. In short, a midline incision was made from the xiphoid to the pubic symphysis. The edges of the incision were retracted bilaterally. Peritonectomy procedures were performed with electroevaporative surgery using a ball-tipped diathermy [6]. Cytoreductive surgery consisted of a series of peritonectomy procedures including: anterior parietal peritonectomy, greater omentectomy with splenectomy, left upper quadrant peritonectomy, right upper quadrant peritonectomy, lesser omentectomy with cholecystectomy, and pelvic peritonectomy with rectosigmoid or total colonic resection [7]. The visceral resections included rectosigmoidectomy, right colectomy, total abdominal colectomy, hysterectomy and small bowel resection. These resections were used at anatomic sites where there was visible evidence of disease. All mesothelioma invasion or entrapment of the resected specimens was documented by histopathologic examination.

\section{Perioperative Intraperitoneal Chemotherapy}

After maximal cytoreduction of peritoneal mesothelioma, the skin edges were elevated on a self-retaining retractor to make a reservoir of the abdomen and pelvis to retain the chemotherapy solution. Intraperitoneal hyperthermic chemotherapy with cisplatin $(50 \mathrm{mg} /$ $\left.\mathrm{m}^{2}\right)$ and doxorubicin $\left(15 \mathrm{mg} / \mathrm{m}^{2}\right)$ was administrated in the operating room at approximately $41.5^{\circ} \mathrm{C}$ in $3 \mathrm{~L}$ of $1.5 \%$ dextrose peritoneal dialysis solution for 90 minutes [8]. The intraperitoneal chemotherapy solution was manually distributed to facilitate uniform distribution and penetration of the chemotherapy into residual tumor.

In the early postoperative period (postoperative day 1 to day 5), paclitaxel at $20 \mathrm{mg} / \mathrm{m}^{2} /$ day was administered into the peritoneal cavity in $1 \mathrm{~L}$ of $1.5 \%$ dextrose peritoneal dialysis solution or $6 \%$ hetastarch solution [9]. Nasogastric suctioning, thoracostomy drainage of the pleural spaces, total parental nutrition, deep venous thrombosis prophylaxis and postoperative pain control were used as indicated in the DMPM clinical pathway.

\section{Histologic Materials}

A comprehensive review of the histopathological features of DMPM was performed by two pathologists, who individually evaluated each case. These two pathologists were aware that all 62 patients in this study had clinical evidence of peritoneal mesothelioma but were masked to all other clinical information. The mean number of specimens taken from separate anatomic sites was [11] +4 per patient. The mean number of slides studied was $20+8$ per patient. The assessment and measurement of the histopathological features were finally determined by the predominant findings throughout the multiple specimens examined in each case. Any differences in the interpretation of these slides were resolved by final consensus between the two pathologists.

The immunohistochemical studies were performed using immunostaining on formalin-fixed, paraffin-embedded sections using the avidin-biotin-peroxidase complex method. Sections were cut 3-4 $\mu . \mathrm{m}$ thick, deparaffinized in xylene, and rehydrated in descending grades (100-70\%) of ethanol. Endogenous peroxidase activity was blocked by a 10 -minute treatment with $3 \%$ hydrogen peroxide in absolute methanol. The primary antibodies used were keratin $5 / 6$ by Boehringer-Mannheim (Indianapolis, IN), 1:25 dilution Calretinin by Zymed Laboratories, Inc. (South San Francisco, CA) pre-diluted by the manufacturer; Ber-EP4 by DakoCytomation California Inc. (Carpinteria, CA) 1:30 dilution; and B72.3 by Signet Laboratories Inc. (Dedham, MA) 1:30 dilution. All cases selected were positive for Calretinin and CK 5/6 and negative for at least two epithelial markers including B72.3 and Ber-EP4 [10].

\section{Histopathologic Parameters}

The nuclear size was determined using red blood cells within the microscopic field of view as a reference diameter of approximately $7 \mu$.m. Magnification of 40x was used during this measurement. The measurement was of the predominant nuclei size found throughout the multiple specimens examined. For this study, nucleus size was categorized into two groups: $\leq 30 \mu$.m versus $>30 \mu . \mathrm{m}$. The $\leq 30 \mu . \mathrm{m}$ diameter nuclei were $\leq$ four times the $\mathrm{RBC}$ diameter; the $>30 \mu$.m diameter nuclei were $>$ four times the RBC diameter. These measurements of the DMPM nuclei were supplemented by a micrometer. The mesothelioma cell differentiation was categorized by using a histological grading system recommended by the American Joint Cancer Commission [11]. It was based on a qualitative assessment of the differentiation of a tumor expressed as the extent to which a tumor resembled the normal tissue of the primary cancer site. It ranged from well-differentiated to moderately-differentiated to poorly-differentiated and was evaluated strictly by architectural appearance of a given neoplasm. DMPM was well-differentiated, if clear recognition of tubular, papillary, cystic, or a combination of these patterns were seen. DMPM was scored as poorlydifferentiated, if tubular, papillary and cystic structures were greatly substituted by solid sheets of tumor. In the moderate-differentiated cases there was a significant loss of the well-differentiated pattern; however poorly-differentiated segments were not so predominant.

DMPM were classified into epithelial, biphasic or sarcomatoid histologic types, according to the World Health Organization classification [12]. The nuclear/cytoplasmic ratio was evaluated at 40x magnification and categorized into normal versus increased nuclear/cytoplasmic ratio. Mitotic count was evaluated in 10 highpower microscopic fields (HPF) at $40 \mathrm{x}$ and categorized into $\leq 4$ versus $>$ 4. Histopathologic features of atypical mitosis, tumor necrosis, pleomorphism, perineural invasion and lymph nodal involvement were designated as absence versus presence. Depth of invasion of visceral specimens was also studied as either superficial or deep. Superficial invasion was classified when the malignant cells limited to the subserosal layer. Deep invasion was classified when tumor cells were evident in the deeper layers of the bowel (i.e. muscularis propria, submucosa and mucosa).

The chromatin pattern of the nucleus was evaluated by the distribution of chromatin material. In normal or reactive mesothelial cells the chromatin pattern was usually uniform and granular. In some cases it was noted that nuclei would have a clear chromatin pattern. The chromatin pattern was classified into two groups: uniform and granular versus clear.

Nucleolar size was determined using a reticle installed in a light microscope and categorized into $\leq 3 \mu$. m versus $>3 \mu$.m.

\section{Statistical Analysis}

Institutional review board approval was obtained to analyze these data. Survival analysis was performed by using Kaplan-Meier method and compared using the log rank test. The correlations of nuclear size and 12 histopathologic parameters were determined by univariate analysis using Chi [2]. (or Fisher's exact) test. All statistical analyses were performed using SPSS for Windows (Version 11.5; SPSS GmbH, Munich, Germany). A significant difference was assumed for a p-value less 0.05 .

\section{Results}

\section{Clinical Data}

There were 34 male patients (55\%). The mean age at the time of 
Citation: Yan TD, Brun EA, Cerruto CA, Chang D, Sugarbaker PH (2012) Analysis of Histologic Parameters Associated with Nuclear Size in Diffuse Malignant Peritoneal Mesothelioma Uniformly Treated by Cytoreductive Surgery and Perioperative Intraperitoneal Chemotherapy. Surgery S8:001. doi:10.4172/2161-1076.S8-001

Page 3 of 5

diagnosis of DMPM was $47+13$ years. The mean age at the time of surgery was $49+13$ years. All patients were followed until October 1 , 2005 or until death. The median follow-up was 37 months (range 8 to 143 months). Thirty-six patients (58\%) were alive at the last time of contact.

\section{Survival Results}

The overall median survival was 79 months (range 1 to 143 months), with 1-, 3- and 5-year survival rates of $84 \%, 58 \%$ and $50 \%$, respectively. The 1-, 3- and 5year survival of patients with nucleus size $\leq 30 \mu$.m were $100 \%, 90 \%$ and $77 \%$, respectively. The 1-, 3- and 5-year survival of patients with nucleus size $>30 \mu$.m were $62 \%, 37 \%$ and $18 \%$, respectively. The prognostic significance of nuclear size for overall survival was $\mathrm{p}<.001$ (Figure 1)

\section{Histopathologic Results}

The mean nuclear size was $3+10 \mu . m$. Thirty-five patients had nuclear size of $\leq 30 \mu . \mathrm{m}$ and 27 patients had nuclear size $>30 \mu . \mathrm{m}$. Table 1 demonstrates the correlations between nuclear size and 12 histopathologic parameters, including cell differentiation, histologic type, nuclear/cytoplasmic ratio, mitotic count, atypical mitosis, tumor necrosis, pleomorphism, perineural invasion, lymph nodal involvement, depth of invasion chromatin pattern and nucleolar size by univariate analysis.

Histologic type $(\mathrm{p}=.012)$ (Figure 2$)$, nuclear/cytoplasmic ratio ( $\mathrm{p}$ $<.001)$ (Figure 3), mitotic count $(\mathrm{p}=.001)$ (Figure 4$)$, atypical mitosis $(\mathrm{p}<.001)$ (Figure 5) and tumor necrosis $(\mathrm{p}<.001)$ (Figure 6) were strongly correlated with nuclear size.

Chromatin pattern and nucleolar size were $100 \%$ correlated with nuclear size. All 35 patients with nuclear size of $\leq 30 \mu . \mathrm{m}$ had uniform/ granular chromatin pattern and nucleolar size of $\leq 3 \mu . \mathrm{m}$ (Figure 7). In contrast all 27 patients with nuclear size $>30 \mu . \mathrm{m}$ had clear chromatin pattern and nucleolar size of $>3 \mu . \mathrm{m}$ (Figure 8 ).

\section{Discussion}

Evolution in the management of DMPM has occurred over the last two decades [11]. Cytoreductive surgery combined with perioperative

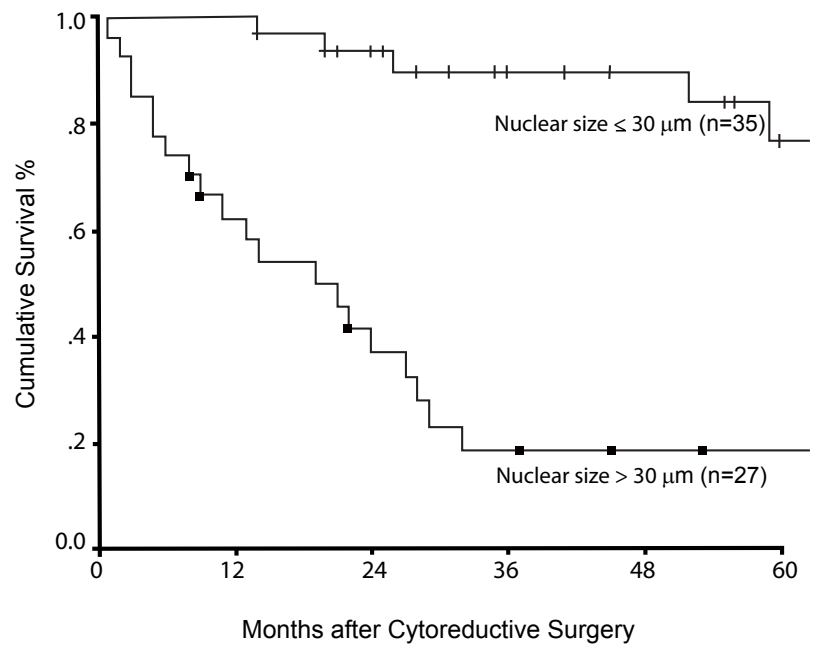

Figure 1: Survival by nuclear size after cytoreductive surgery and perioperative intraperitoneal chemotherapy for patients with diffuse malignant peritoneal mesothelioma $(n=62)$. Prognostic significance of nuclear size was $p$ $<.001$.

\begin{tabular}{|c|c|c|c|}
\hline \multirow[b]{2}{*}{ Variable } & \multicolumn{2}{|c|}{ Nuclear Size } & \multirow{2}{*}{$\begin{array}{l}\mathrm{Chi}^{2} \\
\mathrm{P} \text {-value }\end{array}$} \\
\hline & $\begin{array}{l}\leq 30 \mu \mathrm{m} \\
\mathrm{n}(\%)\end{array}$ & $\begin{array}{l}>30 \mu \mathrm{m} \\
\mathrm{n}(\%)\end{array}$ & \\
\hline Total & $35(100)$ & $27(100)$ & - \\
\hline Cell differentiation & - & - & 1.000 \\
\hline Well/moderately differentiated & $24(69)$ & $19(70)$ & - \\
\hline Poorly differentiated & $11(31)$ & $8(30)$ & - \\
\hline Histologic type & - & - & .012 \\
\hline Epithelial & $35(100)$ & $22(82)$ & - \\
\hline Biphasic & $0(0)$ & $5(18)$ & - \\
\hline Nuclear/cytoplasmic ratio & - & - & $<.001$ \\
\hline Normal & $33(94)$ & $9(33)$ & - \\
\hline Increased & $2(6)$ & $18(67)$ & - \\
\hline Mitotic count & - & - & .001 \\
\hline$\leq 4$ & $34(97)$ & $17(63)$ & - \\
\hline$>4$ & $1(3)$ & $10(37)$ & - \\
\hline Atypical mitosis & - & - & $<.001$ \\
\hline Absent & $35(100)$ & $16(59)$ & - \\
\hline Present & $0(0)$ & $11(41)$ & - \\
\hline Tumor necrosis & - & - & $<.001$ \\
\hline Absent & $31(89)$ & $12(44)$ & - \\
\hline Present & $4(11)$ & $15(56)$ & - \\
\hline Pleomorphism & - & - & .359 \\
\hline Absent & $29(83)$ & $19(70)$ & - \\
\hline Present & $6(17)$ & $8(30)$ & - \\
\hline Perineural invasion & - & - & .279 \\
\hline Absent & $32(91)$ & $22(82)$ & - \\
\hline Present & $3(9)$ & $5(19)$ & - \\
\hline Lymph nodal involvement & - & - & .223 \\
\hline Absent & $33(94)$ & $22(82)$ & - \\
\hline Present & $2(6)$ & $5(18)$ & - \\
\hline Depth of invasion & - & - & .124 \\
\hline Infiltration limited to subserosal layer & $24(69)$ & $13(48)$ & - \\
\hline Infiltration beyond subserosal layer & $11(31)$ & $14(52)$ & - \\
\hline Chromatin Pattern & - & - & $<.001$ \\
\hline Uniform/granular & $35(100)$ & $0(0)$ & - \\
\hline Clear & $0(0)$ & $27(100)$ & - \\
\hline Nucleolar size & - & - & $<.001$ \\
\hline$\leq 3 \mu \mathrm{m}$ & $35(100)$ & $0(0)$ & - \\
\hline$>3 \mu \mathrm{m}$ & $0(0)$ & $27(100)$ & - \\
\hline
\end{tabular}

Table 1: Correlations between nuclear size and 12 other histopathologic parameters.

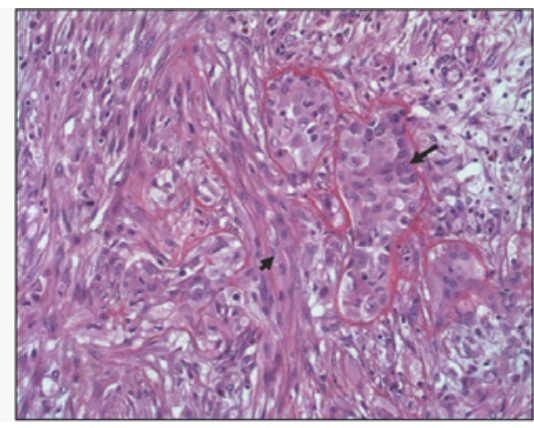

Figure 2: Biphasic type of diffuse malignant peritoneal mesothelioma at 20x shows features of both sarcomatoid (short arrow) and epithelial (long arrow) types, characterized by spindle-looking mesothelial cells within nests of epithelial mesothelial cells. 
Citation: Yan TD, Brun EA, Cerruto CA, Chang D, Sugarbaker PH (2012) Analysis of Histologic Parameters Associated with Nuclear Size in Diffuse Malignant Peritoneal Mesothelioma Uniformly Treated by Cytoreductive Surgery and Perioperative Intraperitoneal Chemotherapy. Surgery S8:001. doi:10.4172/2161-1076.S8-001

Page 4 of 5

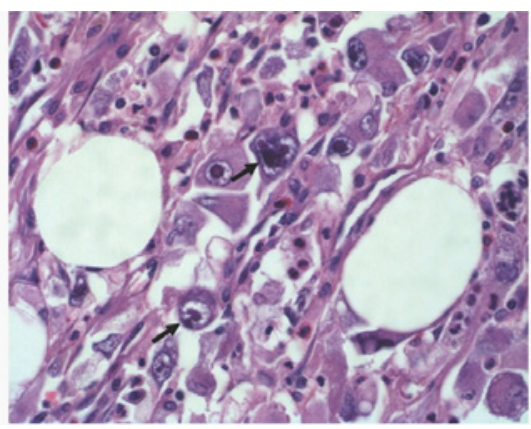

Figure 3: Diffuse malignant peritoneal mesothelioma at 40x shows increased nuclear/cytoplasmic ratio (long arrow).

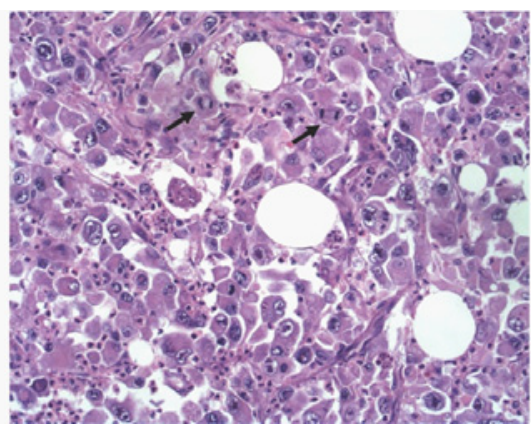

Figure 4: Diffuse malignant peritoneal mesothelioma at 20x shows presence of mitosis (long arrow).

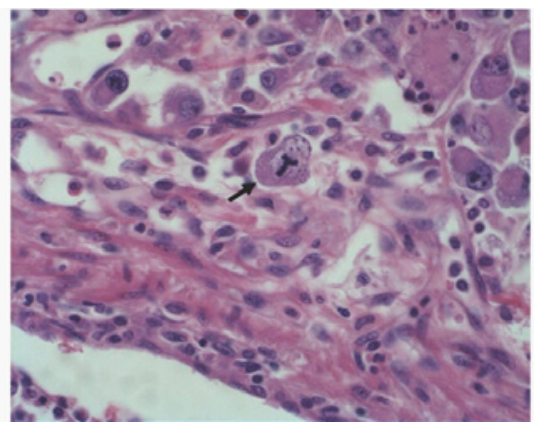

Figure 5: Diffuse malignant peritoneal mesothelioma at 40x shows presence atypical mitosis (long arrow)

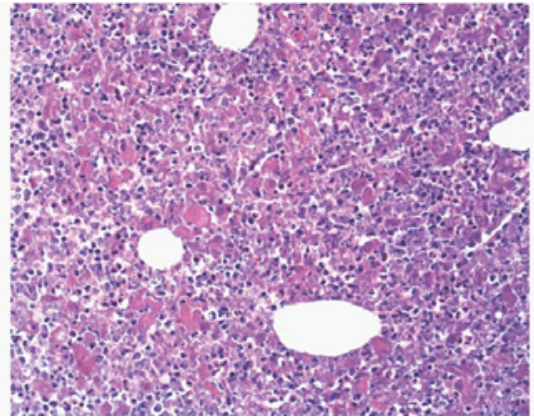

Figure 6: Diffuse malignant peritoneal mesothelioma at 20x shows presence of tumor necrosis.

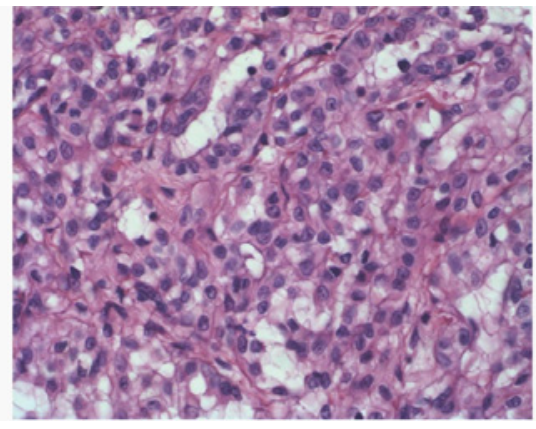

Figure 7: Diffuse malignant peritoneal mesothelioma at 40x shows nuclear size of $\leq 30 \mu \mathrm{m}$, uniform/granular chromatin pattern and nucleolar size of $\leq$ $3 \mu \mathrm{m}$.

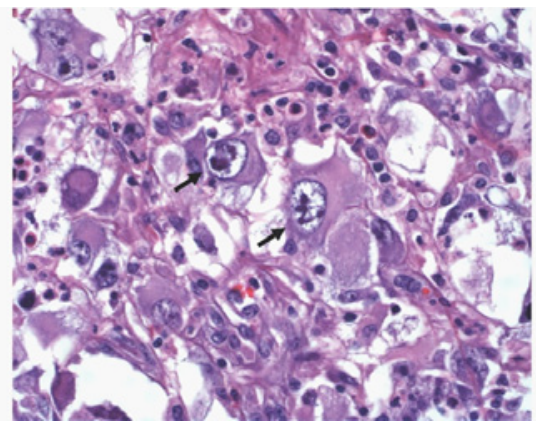

Figure 8: Diffuse malignant peritoneal mesothelioma at 40x shows nuclear size $>30 \mu \mathrm{m}$, clear chromatin pattern and nucleolar size of $>3 \mu \mathrm{m}$ (long arrow).

intraperitoneal chemotherapy is a comprehensive treatment alternative that has become available in recent years. Several international centers have reported an improved survival in selected patients with DMPM who were treated with this combined modality, achieving a median survival of 50 to 90 months [12-16]. As more patients are treated in this uniform manner, a meaningful clinic pathologic study of this rare disease is made possible.

There is a great variation in histopathologic features exists in DMPM. In 1995 a nuclear grading system was first described by Goldblum and Hart based on histomorphologic interpretations of mesothelioma nuclear size, chromatin pattern and nucleolar size [3]. The authors found that tumors with a low-grade nuclei tended to be less aggressive. This interesting observation was further validated by Nonaka and colleagues, who demonstrated that low-grade nuclei were associated with an improved survival in DMPM patients undergoing cytoreductive surgery and perioperative intraperitoneal chemotherapy [4]. However, the nuclear grading system used in this report did not quantitate the nuclear size. Consistent histopathologic grading by different pathologists was difficult. Previously, our group characterized the histomorphologic features of DMPM, but no attempt was made to identify the correlations among these parameters [5].

As our study of the histomorphology of the peritoneal mesothelioma cell continued, it became evident that measurement of the cell nucleus using a micrometer was not consistent. The shrinkage of tissues in different case material varied considerably. Also, there were differences between the fixation of very large tumor specimens received from the operating room (i.e., the greater omentum) and thin specimens (i.e., strips of peritoneum). Also, we found that micrometers on different light microscopes were difficult to calibrate in exactly the 
Citation: Yan TD, Brun EA, Cerruto CA, Chang D, Sugarbaker PH (2012) Analysis of Histologic Parameters Associated with Nuclear Size in Diffuse Malignant Peritoneal Mesothelioma Uniformly Treated by Cytoreductive Surgery and Perioperative Intraperitoneal Chemotherapy. Surgery S8:001. doi:10.4172/2161-1076.S8-001

Page 5 of 5

same manner. Finally, adding to our difficulties was the fact that only a few light microscopes used in the reading of these slides were equipped a micrometer. After considerable rereading of slides and discussion, the red cell nucleus was retained as the standard of measurement for this nuclear grading scale.

There was a second change in our standard procedures for the use of nuclear size in assessing prognosis as compared to the original manuscript by Cerruto et al. [5]. Our original report utilized four sizes of nucleus for prognostication. In this report the original Class I and Class II nuclei are combined into a Group I. The classification III and IV were placed together into a Group II. This simplification of the reading of nuclear size was designed to provide an easier assessment (estimation) for utilization of this information by the general pathologist.

Although nuclear size has also been proposed as a staging system in other malignancies such as renal cell carcinoma and breast cancer, the assessment of nuclear size in DMPM is unfamiliar to most pathologists and the correlations of nuclear size with other histopathologic parameters has not been previously studied. With a hypothesis that nuclear size might reflect the biological behavior of DMPM, we performed a statistical assessment of the correlations between nuclear size and 12 histopathologic parameters.

These data showed $100 \%$ correlation of nuclear size with chromatin pattern and nucleolar size. All 35 patients with nuclear size of $\leq 30$ $\mu . \mathrm{m}$ had uniform/granular chromatin pattern and nucleolar size of $\leq 3 \mu$.m. In contrast, all 27 patients with nuclear size $>30 \mu . \mathrm{m}$ had clear chromatin pattern with or without intracellular inclusions and nucleolar size of $>3 \mu \mathrm{m}$. These findings show that there is a remarkable consistency between nuclear size, chromatin pattern and nucleolar size in DMPM. These data statistically supports the original nuclear grading system proposed by Goldblum and Hart [3]. These data confirm that a nuclear grading system that includes nuclear size, chromatin pattern, and nucleolar size is of value in a histologic assessment of prognosis in this disease DMPM.

It showed that nuclear size $>30 \mu$.m was strongly associated with biphasic histologic type, increased nuclear/cytoplasmic ratio, $>4$ mitotic count, presence of atypical mitosis, presence of tumor necrosis, clear chromatin pattern and nucleolar size $>3 \mu . \mathrm{m}$.

Three studies have demonstrated that increased mitotic count was associated with a less favorable prognosis in DMPM patients $[4,17,18]$. Also, the presence of increased nuclear/cytoplasmic ratio, atypical mitosis and tumor necrosis is correlated with the nuclear size and could be used to predict survival. However, the correlations with nuclear size in these parameters were not as perfect as chromatin pattern and nuclear size. As suggested by Cerruto and colleagues, these parameters could be used as minor prognostic criteria.

Although much progress has been made, greater insight regarding the clinical implications of these findings is required. Since such distinct survival differences are recognized, modification of treatment plans for patients with a high nuclear grade is warranted. For example, neoadjuvant systemic chemotherapy with pemetrexed and cisplatin may be indicated in this less favorable group prior to initiating the local-regional treatment option with cytoreductive surgery and perioperative chemotherapy.

In summary, DMPM Grade I nucleus is seen as a favorable prognosis with a median survival that was not reached at 5 years. The Group I nuclei are $\leq 30 \mu . \mathrm{m}$ in diameter $(\leq 4 \mathrm{x}$ the RBC diameter seen in the field of view). An adjunctive finding to be expected in a study of this clinical method is a nucleolus $\leq 3 \mu . \mathrm{m}(<$ half the RBC diameter) and chromatin of a granular pattern. The Group II nuclei are seen in poor prognosis patients with a median survival of approximately 20 months. The nuclear size of $>30 \mu . \mathrm{m}$ in diameter $(>4 \mathrm{x}$ the RBC diameter seen in the field of view) was associated with the nucleolus of $>3 \mu . \mathrm{m}(<$ half the $\mathrm{RBC}$ diameter) and has a clear nuclear chromatin.

\section{References}

1. Kerrigan SA, Turrnir RT, Clement PB, Young RH, Churg A (2002) Diffuse malignant epithelial mesotheliomas of the peritoneum in women: A clinicopathologic study of 25 patients. Cancer 94: 378-385.

2. Battifora H, McCaughey W (1994) Tumors of the Serosal Membranes. Washington, DC (USA): Armed Forces Institute of Pathology.

3. Goldblum J, Hart WR (1995) Localized and diffuse mesotheliomas of genita tract and peritoneum of women. A clinicopathologic study of nineteen true mesothelial neoplasms, other than adenomatoid tumors, multicystic mesotheliomas, and localized fibrous tumors. Am J Surg Pathol 19: 1124-1137.

4. Nonaka D, Kusamura S, Baratti D, Casali P, Cabras AD, et al. (2005) Diffuse malignant mesothelioma of peritoneum. Cancer 104: 2181-2188.

5. Cerruto CA, Brun EA, Chang D, Sugarbaker PH (2006) Prognostic significance of histomorphologic parameters in diffuse malignant peritoneal mesothelioma. Arch Pathol Lab Med 130: 1654-1661.

6. Sugarbaker PH (1994) Dissection by electrocautery with a ball tip. J Surg Onco 56: $246-248$.

7. Sugarbaker PH (1995) Peritonectomy procedures. Ann Surg 221: 29-42.

8. Sugarbaker PH, Welch LS, Mohamed F, Glehen O (2003) A review of peritoneal mesothelioma at the Washington Cancer Institute. Surg Oncol Clin N Am 12: 605-621.

9. Mohamed F, Marchettini P, Stuart OA, Sugarbaker PH (2003) Pharmacokinetics and tissue distribution of intraperitoneal paclitaxel with different carrier solutions. Cancer Chemother Pharmacol 52: 405-410.

10. Riera JR, Astengo-Osuna C, Longmate JA, Battifora H (1997) The immunohistochemical diagnostic panel for epithelial mesothelioma. A reevaluation after heat-induced epitope retrieval. Am J Surg Pathol 21: 14091419.

11. Greene FL, Page DL, Fleming ID, et al. (2002) AJCC Cancer Staging handbook from the AJCC Cancer Staging Manual. Sixth Edition. New York, NY (USA): Springer.

12. Weiss SW (1994) World Health Organization, International Histological Classification of Tumors. Histological typing of soft tissue tumors. Second Edition. Berlin, Springer-Verlag.

13. Deraco M, Nonaka D, Baratti D, Casali P, Rosai J, et al. (2006) Prognostic analysis of clinicopathologic factors in 49 patients with diffuse malignant peritoneal mesothelioma treated with cytoreductive surgery and intraperitoneal hyperthermic perfusion. Ann Surg Oncol 13: 229-237.

14. Brigand C, Monneuse O, Mohamed F, Sayag-Beaujard AC, Isaac S, et al (2006) Malignant peritoneal mesothelioma treated by cytoreductive surgery and intraperitoneal chemohyperthermia: Results of a prospective study. Ann Surg Oncol 13: 405-412.

15. Feldman AL, Libutti SK, Pingpank JF, Bartlett DL, Beresnev TH, et al. (2003) Analysis of factors associated with outcome in patients with malignan peritoneal mesothelioma undergoing surgical debulking and intraperitoneal chemotherapy. J Clin Oncol 21: 4560-4567.

16. Loggie BW, Fleming RA, McQuellon RP, Russell GB, Geisinger KR, et al (2001) Prospective trial for the treatment of malignant peritoneal mesothelioma. Am Surg 67: 999-1003.

17. Beer TW, Carr NJ, Whittaker MA, Pullinger N (2004) Mitotic and in situ end labeling apoptotic indices as prognostic markers in malignant mesothelioma. Ann Diag Pathol 4: 143-148.

18. Ramael M, Jacob W, Weyler J, Van Meerbeeck J, Białasiewicz P, et al. (1994) Proliferation in malignant mesothelioma as determined by mitosis counts and immunoreactivity for proliferating cell nuclear antigen (PNCA). J Pathol 172 247-253.

This article was originally published in a special issue, Peritoneal Mesothelioma- Symptoms, Diagnosis and Therapy; Surgical procedures for Hyperhidrosis; Hepatobiliary: Diagnosis and Therapy handled by Editor(s). Dr. Roberto Gedaly, University of Kentucky College of Medicine, USA 